\title{
Middle Ear Carcinoma In Situ
}

National Cancer Institute

\section{Source}

National Cancer Institute. Middle Ear Carcinoma In Situ. NCI Thesaurus. Code C91741.

An intraepithelial carcinoma of the middle ear without evidence of invasion. 